J. Clin. Chem. Clin. Biochem.

Vol. 20, 1982, pp. 851-859

\title{
Ergebnisse aus Ringversuchen für Harnsteinanalysen
}

Von G. Röhle,

Institut für Klinische Biochemie der Universität Bonn

U. Voigt,

Institut für Medizinische Statistik, Dokumentation und Datenverarbeitung der Universität Bonn

A. Hesse,

Harnsteinforschungsstelle der Urologischen Universitätsklinik Bonn und

H. Breuer ${ }^{1}$ )

Institut für Klinische Biochemie der Universität Bonn

(Eingegangen am 14. Juli/26. August 1982)

Zusammenfassung: Im Rahmen der Externen Qualitätskontrolle der Deutschen Gesellschaft für Klinische Chemie wurden in den Jahren 1980 und 1981 vier Ringversuche für Harnsteinanalysen durchgeführt. In diesen Ringversuchen erhielten die teilnehmenden Laboratorien insgesamt 16 Proben (acht Reinsubstanzen und acht Gemische mit zwei Komponenten), die qualitativ und quantitativ zu analysieren waren. Als qualitative Analysenergebnisse konnten vereinfachte Beschreibungen der vorliegenden Analyte - z.B. unter Vernachlässigung der Zahl von Kristallwassermolekülen - angegeben werden oder, soweit es die angewendete Analytik zuließ̧, die exakte chemische Zusammensetzung der Sụbștanzen. Von 1283 durchgefürten Analysen erreichten 818 (64\%) das vereinfachte Analysenziel. Die häufigsten Fehler chemischer Analysen waren falsch negative und falsch positive Ergebnisse für Calciumoxalat sowie falsch negative Ergebnisse von Calciumphosphat. Auch den Anwendern der Infrarotspektroskopie unterliefen bei diesen Bestandteilen die meisten Fehlanalysen. Die żuverlässigsten Ergebnisse wurden mit der Röntgendiffraktion ermittelt; $86 \%$ der Analysen erreichten fehlerfrei das differenzierte Analysenziel. Die insgesamt nahezu fehlerfreien Resultate einiger Teilnehmer, die mit chemischer Analyse arbeiteten, lassen jedoch den Schluß zu, daß neben dem angewendeten Analysenprinzip die Qualifikation des Analytikers von entșcheidender Bedeutung für die Zuverlässigkeit der Ërgebnisse ist.

\section{Results of quälity control surveys for urinary calculus analyses}

Summary: In 1980 and 1981, four quality control surveys for urinary calculus analyses were carried out, within the framework of external quality controls of the Deutsche Gesellschaft für Klinische Chemie. During these quality control surveys the participating laboratories received a total of 16 samples (eight pure substances and eight mixtures of two components), to be analyzed qualitatively and quantitatively. For the qualitative analyses, a laboratory could provide simplified descriptions of the analytes - for example, without stating the number of molecules of the water of crystallization; or, to the extent permitted by the mode of analysis, the exact chemical composition could be presented. Of 1283 analyses, 818.(64\%) attained the goals of the simplified analysis. The most frequent errors in chemical analyses were false negative and false positive results for calcium oxalate, and false negative results for calcium phosphate. Laboratories using analysis by infrared spectroscopy also submitted the biggest proportion of incorrect analyses for these components. The most reliable results were produced by X-ray-diffraction: $86 \%$ of the results in this category represented correct differentiated analyses. The generally accurate results achieved by some participants using chemical analysis suggest, however, that - in addition to the principle of analysis used - the qualification of the operator is an important determinant in the reliability of the results.

\footnotetext{
1) Prof. Dr. H. Breuer, Mitherausgeber unserer Zeitschrift, verstarb am 20. August 1982.
} 


\section{Einführung}

In den meisten Ländern, in denen moderne klinische Chemie betrieben wird, sind Ringversuche als Maßnahme zur externen Qualitätssicherung der Zuverlässigkeit von Analysenergebnissen seit längerem eingeführt. Hauptsächlich sind quantitative klinisch-chemische Bestimmungen der Bestandteile von Körperflüssigkeiten Gegenstand dieser Ringversuche, seltener qualitative Analysen. Uber Ringversuche für Harnsteinanalysen liegen bisher nur wenige Untersuchungen vor $(1,2)$, obwohl die Diagnostik der Urolithiasis für die Patientenversorgung eine sehr große Bedeutung hat. Nach jüngeren epidemiologischen Untersuchungen haben z. B. in der Bundesrepublik Deutschland 50 von 1000 Personen Harnsteinleiden, deren Behandlung entscheidend von den Ergebnissen der Analysen der Biomineralisate abhängt (3).

Der Grund für die bisherige Vernachlässigung der Qualitätssicherung von Harnsteinanalysen liegt unter anderem sicherlich in der komplexen Struktur des in der Harnsteinanalytik anfallenden Datenmaterials, das mit den üblichen Auswertungsprogrammen für quantitative Analysenergebnisse nicht zu bewältigen ist. Bei der Auswertung von Ringversuchen für Harnsteinanalysen ist neben der Ja-Nein-Entscheidung, ob ein Probenbestandteil richtig identifiziert wurde, die große Zahl der Möglichkeiten von Falschanalyșen (Idẹtifizierung von Substanzen, die in einer Probe tatsächlich nicht vorliegen) in Rechnung zu stellen. Es ist zu berücksichtigen, daß die Anwendung verschiedener Analysentechniken zu Ergebnissen führen kann, die untereinander nur schwer zu vergleichen sind. So ermöglicht die chemische Analyse nur den Nachweis einzelner Ionen, und die Identifizierung der ursprünglichen Probenzusammensetzung erfolgt durch Rückschluß. Andererseits werden durch physikalische Analysentechniken wie Infrarotspektroskopie und Röntgendiffraktion die Analysensubstanzen nicht verändert und man erhält differenziertere Informationen über ihre Zusammensetzung, z. B. den Kristallwassergehalt der Moleküle. Soweit die Analysenproben aus Substanzgemischen bestehen, muß in der Auswertung die Unterscheidung von Haupt- und Nebenbestandteilen dokumentiert werden. Es ist weiterhin festzuhalten, wieviele Ergebnisse richtig, falsch oder in welchem $\mathrm{Maß}$ partiell richtig sind. Schließlich müssen quantitative Ergebnisse - soweit sie vorliegen - dargestellt werden.

\section{Nachdem ein Auswertungsprogramm entwickelt} worden war, das diese vielfältigen Zusammenhänge weitgehend berücksichtigt, wurden in Zusammenarbeit mit der Deutschen Gesellschaft für Klinische Chemie 1980 und 1981 jeweils żwei Ringversuche für Harnsteinanalysen durchgeführt, an denen sich Laboratorien aus der Bundesrepublik Deutschland und aus anderen europäischen Ländern beteiligten.

\section{Material und Methoden}

\section{Proben}

In den Ringversuchen der Jahre 1980 und 1981 wurden 16. verschiedene Proben eingesetzt, deren Bestandteile in der Tạbelle 1 aufgefuhrt sind. Jeweils zwei Proben jedes Ringversuchs waren Reinsubstanzen, zwei weitere hatten zwei Beștandteile. Bei den Proben 3A, 4B und 4C handelte es sich um homogenisierte Harnsteine von Patienten. Die Probe $2 \mathrm{~B}$ (Monoammoniumurat) stellte Prof. Klee, Karlsruhe, zur Verfigung. Alle übrigen Proben wurden aus Reinsubstanzen hergestellt, die im Handel erhältlich sind: Apatit (Fluka A.G.), Brushit (Merck, Nr. 2146), Cystin (Merck, Nr. 2837), Harnsäure (Flukka A.G.), Struvit (R̈iedël de Haën), Whewellit (Roth, Karlsruhe).

\section{Ringversuche}

Es wurden vier Ringversuche zu folgenden Termininen dựchgeführt: April 1980, November 1980, März 1981 und Oktober

1981. Bei jedem Ringversuch erhielt jedes teilnehmeñde Laboratorium vier verschiedene Proben, die innerhalb einer Woche żu analysieren waren. Mit den Proben erhielten die Tèilnehmier eine Liste der potentiellen Probenbestandteile, in der einerseits die genauen Bezeichnungen der Substanzen und andererseits - wegen der eingeschränkten Leistungsfähigkeit einiger Analy sentechniken - vereinfachte Analysenziele angegeben waren (Tab. 2). In dieser Liste wurden auch die in der Harnsteinanaly tik vorkommenden Artefakte Cholesterin, Calcit, Gips und Quarz berücksichtigt.

Als Analysenergebnisse konnten die gefundenen Einzelbestandteile (Ionen) angegeben werden; es sollten jedoch immer Rückschlüsse auf die in den Proben enthaltenen Substanzen gezogen werden; möglichst war die genaue Bezeichnung anżugeben, andernfalls das vereinfachte Analysenziel. Vereinbarungsgemäß bestanden die Proben aus höchstens zwei Substạnżeñ. Soweit mit der qualitativen Analyse in einer Probe zwei Substanzen gefunden wurden, war zụsätzlich nach deren Massenverhältnissen gefragt; zumindest aber war zwivischen Hauptund Nebenbestandteil zu unterșcheiden.

\begin{abstract}
Analysenziele
Die qualitativen und quantitativen Analy senziele der Proben $3 A, 4 B$ und $4 C$ wurden vor den Ringversuchen auf der Basis von Analysenergebnissen festgelegt, die vön Referenzlaboratorien ermittelt worden waren. Bei allen anderen Probèn waren die Analysenziele durch die verwendeten Reinsubstanzen bzw. deren Mischungsverhältnisse vorgegeben.
\end{abstract}

\section{Aụswertung der Ergebnisse}

Ergebnisse von Teilnehmern, die lediglich die gefundenen Einzelbestandteile (Ionen) angegeben hatten, wurden in dieser Auswertüng nicht berücksichtigt.

Ebenso entfiel hier die Bewertung der Unterscheidung zwischen Haupt- und Nebenbestandteilen bei Subștanzgemischen.

\section{Definitionen}

Optimales qualitatives Analysenziel: Identifizierung der in einer Probe vorliegenden Substanzen.

Optimales quantitatives Analysenziel: Ermittlung der richtigen Massenverhältniszahlen $\pm 0,2$ bei optimalem qualitativen Ergebnis für Proben mit zwei Bestạndeiden.

Vereinfachtes qualitatives Analysenziel (Tab. 2):Angenäherte Identifizierung der in einer Proobé vóriliegenden Substanzen, soweit es mit den heute in der $\overrightarrow{\mathrm{R}}$ outineanalytik gebräuchlichen chemischen Nachweismethoden möglich ist.

Vereinfachtes quantitatives Analysenziel: Ermittlung dẹ richtigen Massenverhälțniszahlen $\pm 0,2$ bei,erreichtem vereinfachten Analysenziel für Proben mit zyei Beștạdteilen. 


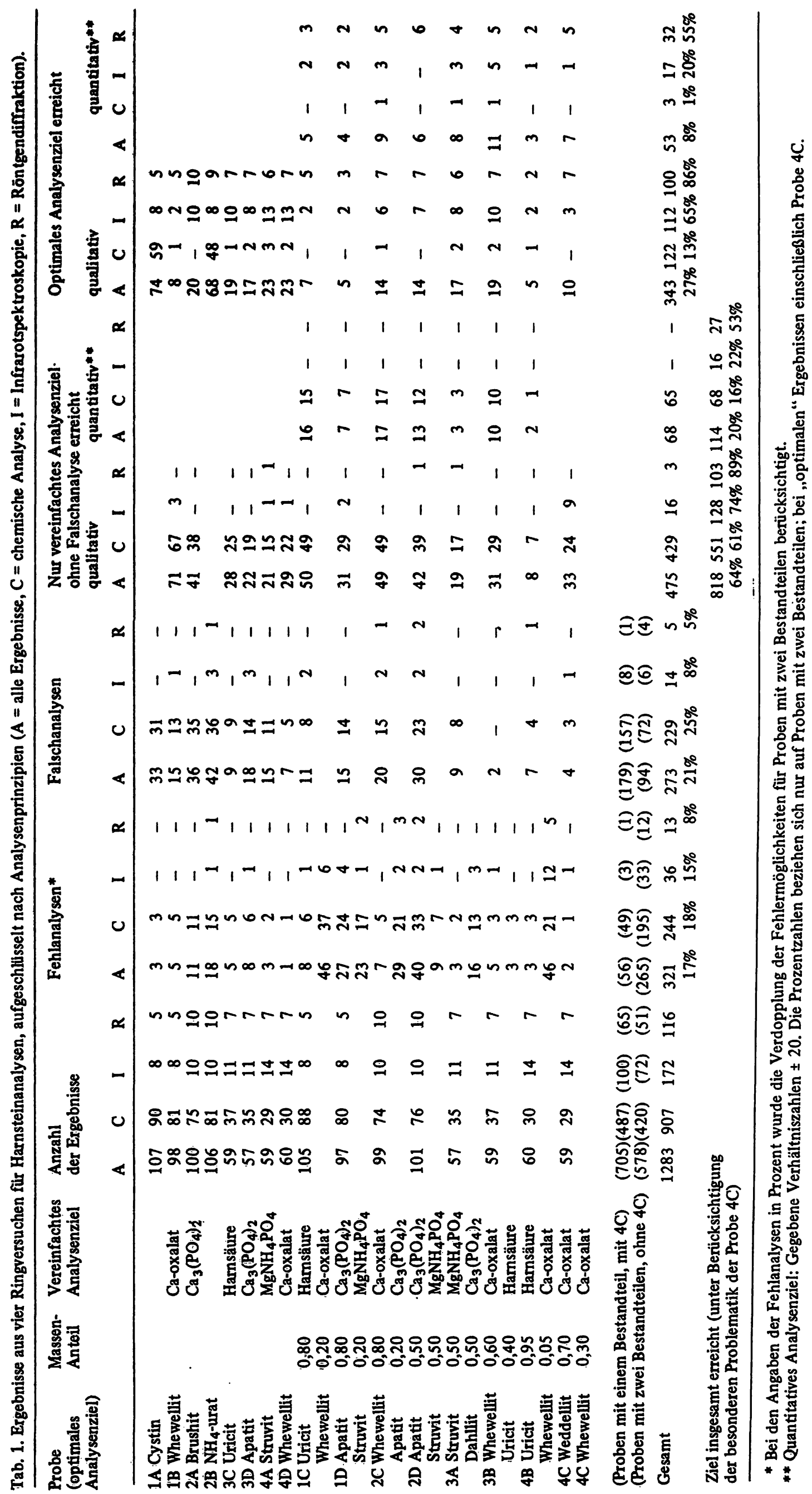


Fehlanalyse: Ein in der Probe vorliegender Bestandteil wurde - auch bei Zugrundelegung des vereinfachten Analysenziels - nicht identifiziert.

Falschanalyse: Es wurde eine Substanz gefunden, die nicht in der Probe vorlag.

\section{Korrekturen}

Gegenüber den Erstauswertungen, welche die Ringversuchsteilnehmer erhielten, wurden nach kritischer Überprüfung einige Korrekturen vorgenommen. U.a. war es notwendig, die Ergebnisse „Dahllit" und „Whitlockit" für den Bestandteil Apatit der Probe 1D sowie die Ergebnisse "Whitlockit" und „Apatit" für den Bestandteil Dahllit der Probe 3A als richtig zu bewerten, da eine absolut sichere Festlegung des Analysenziels in diesen Fällen nicht möglich war.

Tabellen

i

In den Tabellen 1, 3 und 4 wurde auf eine konsequente Nomenklatur für die vereinfachten Analysenziele bzw. falsch gefundenen Bestandteile zugunsten kurzer Bezeichnungen und damit besserer Übersichtlichkeit verzichtet.

Tab. 2. Potentielle Bestandteile der Ringversuchsproben. Das „,vereinfachte Analysenziel“ berücksichtigt die eingeschränkten Differenzierungsmöglichkeiten einiger Analysenmethoden.

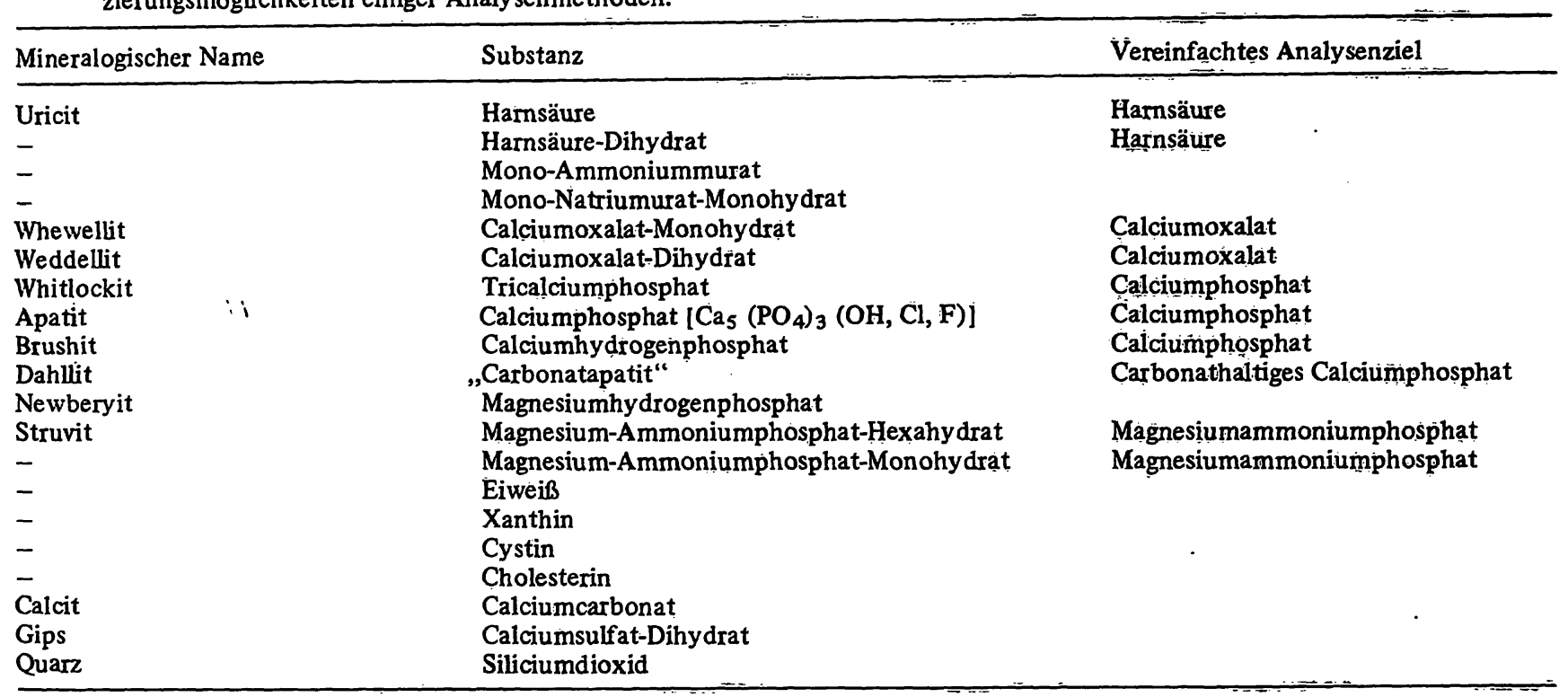

\section{Ergebnisse und Diskussion}

Die Anzahl der Teilnehmer an den vier Ringversuchen und der Anteil derjenigen, die bestimmte Analysenprinzipien angewendet haben, gibt die Tabelle 3 wieder. Die Zahlen unter „Andere Methode“ repräsentieren in erster Linie Teilnehmer, die versäumt haben, Angaben über das angewendete Analysenprinzip zu machen. Insgesamt wurden 1283 Analysen durchgeführt. Deren Ergebnisse sind für alle untersuchten Proben in der Tabelle 1 zahlenmäßig zusammengefaßt. Insgesamt wurde in 321 Fällen (17\%) ein Probenbestandteil nicht gefunden und das vereinfachte Analysenziel somit nicht erreicht. Die Häufigkeit von Fehlanalysen hing wesentlich von der Probensubstanz ab (Tab. 4).
Von großem Einfluß war auch, ob die Proben aus einer oder aus zwei Substanzen bestanden; weiterhin spielten bei Proben mit zwei Substanzen deren Mischungsverhältnisse eine entscheidende Rolle.

Vergleichsweise niedrig ist der Anteil von Fehlanalysen für Cystin (3\%) und Harnsäure (7\%). Besoondere Schwierigkeiten bereitet vielen Analytikern die İdentifizierung von Calciumphosphat, das bei 511 Analysen von Proben, die diese Substanz enthielten, 131 mal (26\%) nicht gefunden wurde. Hoch war der Anteil von Fehlanalysen für Ammoniumurat (17\%), das in erster Linie fälschlich als Harnsäure identifiziert wurde. Sehr groß wär auch die relative Häufigkeit

Tab. 3. Zahl der Teilnehmer an den Ringiversuchen für Harnsteinänạlysen von 1980 bis 1981 , aufgeschlüsselt nach angeweñideten Analysenprinzipien.

\begin{tabular}{|c|c|c|c|c|c|c|}
\hline Ringversuch & $\begin{array}{l}\text { Teilnehmer- } \\
\text { zahl }\end{array}$ & $\begin{array}{l}\text { Chemische } \\
\text { Analyse }\end{array}$ & $\begin{array}{l}\text { Infrarot- } \\
\text { spektroskopie }\end{array}$ & $\begin{array}{l}\text { Röntgen- } \\
\text { diffraktion }\end{array}$ & $\begin{array}{l}\text { Polarisations- } \\
\text { mikroskopie }\end{array}$ & $\begin{array}{l}\text { Andere } \\
\text { Mẽthode }\end{array}$ \\
\hline $\begin{array}{l}\text { April } 1980 \\
\text { November } 1980 \\
\text { März } 1981 \\
\text { Oktober } 1981\end{array}$ & $\begin{array}{r}114 \\
115 \\
61 \\
65\end{array}$ & $\begin{array}{r}96 \\
88 \\
.39 \\
32\end{array}$ & $\begin{array}{r}8 \\
10 \\
11 \\
14\end{array}$ & $\begin{array}{r}5 \\
10 \\
7 \\
8\end{array}$ & $\begin{array}{l}2 \\
1 \\
1 \\
1\end{array}$ & $\begin{array}{r}3 \\
4 \\
3 \\
10\end{array}$ \\
\hline
\end{tabular}


Tab. 4. Anteil der Fehlanalysen für die Bestandteile von 16 Ringversuchsproben gegenüber der sich aus der Zahl der Analysen ergebenden Möglichkeiten zur Fehlanalyse ( $A=$ alle Ergebnisse, $C=$ chemische Analyse, $I=$ Infrarotspektroskopie, $\mathbf{R}=$ Röntgendiffraktion).

\begin{tabular}{|c|c|c|c|c|c|c|c|c|c|c|c|c|}
\hline \multirow{2}{*}{$\begin{array}{l}\text { Probenbestandteil } \\
\text { (vereinfachtes } \\
\text { Analysenziel) }\end{array}$} & \multicolumn{4}{|c|}{ Möglichkeiten } & \multicolumn{4}{|c|}{$\begin{array}{l}\text { Fehlanalysen } \\
\text { absolut }\end{array}$} & \multicolumn{3}{|c|}{ relativ (\%) } & \multirow[b]{2}{*}{$\mathbf{R}$} \\
\hline & $\mathbf{A}$ & C & I & $\mathbf{R}$ & $\mathbf{A}$ & C & I & $\mathbf{R}$ & $\mathbf{A}$ & $\mathrm{C}$ & I & \\
\hline $\begin{array}{l}\mathrm{Ca}_{3}\left(\mathrm{PO}_{4}\right)_{2} \\
\mathrm{Ca}-\mathrm{Oxalat} \\
\mathrm{MgNH}_{4} \mathrm{PO}_{4} \\
\text { Harnsäure } \\
\mathrm{NH}_{4} \text {-urat } \\
\text { Cystin }\end{array}$ & $\begin{array}{l}511 \\
540 \\
314 \\
283 \\
106 \\
107\end{array}$ & $\begin{array}{r}375 \\
369 \\
220 \\
192 \\
81 \\
90\end{array}$ & $\begin{array}{r}60 \\
79 \\
43 \\
43 \\
10 \\
8\end{array}$ & $\begin{array}{r}49 \\
48 \\
29 \\
26 \\
10 \\
5\end{array}$ & $\begin{array}{r}131 \\
112 \\
38 \\
19 \\
18 \\
3\end{array}$ & $\begin{array}{r}108 \\
73 \\
28 \\
17 \\
15 \\
3\end{array}$ & $\begin{array}{r}12 \\
20 \\
2 \\
1 \\
1 \\
-\end{array}$ & $\begin{array}{l}5 \\
5 \\
2 \\
-1 \\
-\end{array}$ & $\begin{array}{r}26 \\
21 \\
12 \\
\cdot 7 \\
17 \\
3\end{array}$ & $\begin{array}{r}29 \\
20 \\
13 \\
9 \\
19 \\
3\end{array}$ & $\begin{array}{r}20 \\
25 \\
5 \\
0 \\
10 \\
0\end{array}$ & $\begin{array}{r}10 \\
10 \\
7 \\
0 \\
10 \\
0\end{array}$ \\
\hline
\end{tabular}

von Fehlanalysen für Calciumoxalat (21\%); diese traten jedoch fast ausschließlich bei den Proben 1C und 4B auf, deren Gehalt an Calciumphosphat sehr gering war. Für die Proben 1B und 4D - jeweils reines Calciumoxalat - waren nur wenige Fehlanalysen zu registrieren.

Generell war die Häufigkeit von Fehlanalysen bei Substanzgemischen deutlich erhöht. Bei 705 Analysen von Reinsubstanzen (einschließlich Probe $4 C$ ) unterliefen $56(8 \%)$ Fehlanalysen, während 578 Untersuchungen von Proben mit zwei Bestandteilen (zweimal 578 Möglichkeiten) zu 265 (23\%) Fehlanalysen führten.

Falschanalysen - die Identifizierung von Bestandteilen, welche in der jeweiligen Probe nicht vorlagen - waren etwa ebenso häufig festzustellen wie Fehlanalysen (Tab. 1). Die Zahl der Falschanalysen wäre wahrscheinlich größer, wenn die Möglichkeit hierzu durch die Versuchsanordnung nicht begrenzt worden wäre, indem für jede Probe nur bị zu zwei gefundene Bestandteile angegeben werden konnten. Für diese Annahme spricht, daß 705 Analysen von Proben mit einem Bestandteil zu 171 (24\%) Falschanalysen, 578 Untersuchungen von Substanzgemischen indessen nur zu 94 (16\%) Falschanalysen führten, obwohl gerade bei den schwieriger zu analysierenden Gemischen eine höhere Fehlerwahr: scheinlichkeit unterstellt werden darf.

Das häufigste Ergebniis von Falschanalysen war Calciumoxalat (Tab. 5). Diese Substanz wurde mit der chemischen Anälyse in allen Proben, in denen sie tatsächlich nicht enthalten war, gefunden; und zwar in $16 \%$ der möglichen Fälle. Der Teil falsch positiver Bestimmungen war besonders hoch, wenn es sich bei einem Probenbestandteil um eine Calciumverbindung handelte. Die starke Tendenz zur Falschanalyse von Calciumoxalat mit chemischen Methoden ist wahrscheinlich auch der Grund dafuir, daß es neun von 30 Analytikern mit chemischen Methoden paradoxerweise gelang, den geringen Gehalt der Probe 4B nachzuweisen, während dies den Teilnehmern, denen die Infrarotspektroskopie oder die Röntgendiffraktion zur Verfügung stand, nur vereinzelt möglich war.
Auffallend groß ist auch die Zahl der Falschanalysen von Harnsäure bei der Probe 1A (Cystin), vor allem aber bei der Probe 2B (Ammoniumurat), im letzteren Fall bedingt durch Fehlanalysen von Ammonium. Allgemein ist zu beobachten, daß die Ergebnisse der meisten Falschanalysen teilweise mit den in den Proben enthaltenen Einzelbestandteilen (Ionen) korrelieren. Wahrscheinlich führte in den Proben vorliegende(s)

- Calcium zu den Falschanalysen Calciumphosphat, Calciumcarbonat oder Calciumsulfat,

- Phosphat zu den Falschanalysen Calciumphosphat, Magnesiumammoniumphosphat oder Magnesiumhydrogenphosphat,

- Ammonium zu den Falschanalysen Magnesiumammoniumphosphat oder Ammoniumurat,

- Harnsäure zu der Falschanalyse Ammoniumurat.

Fälschlich identifiziertes Carbonat dürfte die häufigen Falschanalysen von Carbonatapatit und Calciumcarbonat mitverursacht haben. Andererseits sind wahrscheinlich solche Fälle, in denen entgegen der gegebenen Probenzusammensetzung Xanthin oder Cystin identifiziert wurden, zufälligen Fehlern der Analytiker zuzuschreiben.

Der großen Zahl der festgestellten Fehl- und Falschanalysen entspricht, daß nur $64 \%$ der insgesamt durchgeführten Analysen das vereinfachte qualitative Analysenziel erreichten, ohne daß zugleich eine Falschanalyse unterlief. Mit der chemischen Analyse gelang dies nur in $551(61 \%)$ von 907 Fällen.

Einerseits dürften hierfür Schwächen der Methodik verantwortlich sein: z.B. kann aus der besonders häufig unterlaufenen Falschanalyse von Calciumoxalat geschlossen werden, daß bei Anwendung der üblichen Analysenmethoden die Gefahr von Fehlinterpretationen zu groß ist. Andererseits sind solche Nachteile für den engagierten und erfahrenen Analytiker anscheinend überwindbar. Ein Hinweis darauf ergibt sich aus dem Verhältnis der Zahl der Analysen, mit denen das vereinfachte Analysenziel erreicht wurde, und der Zahl der von einem 
Tab. 5. Zahl der Falschanalysen bei gegebenem Probenmaterial gegenüber der Zahl der Möglichkeiten falsch zu analysieren ( $C$ = chemische Analyse, I = Infrarotspektroskopie, $R=$ Röntgendiffraktion, A = alle Ergebnisse).

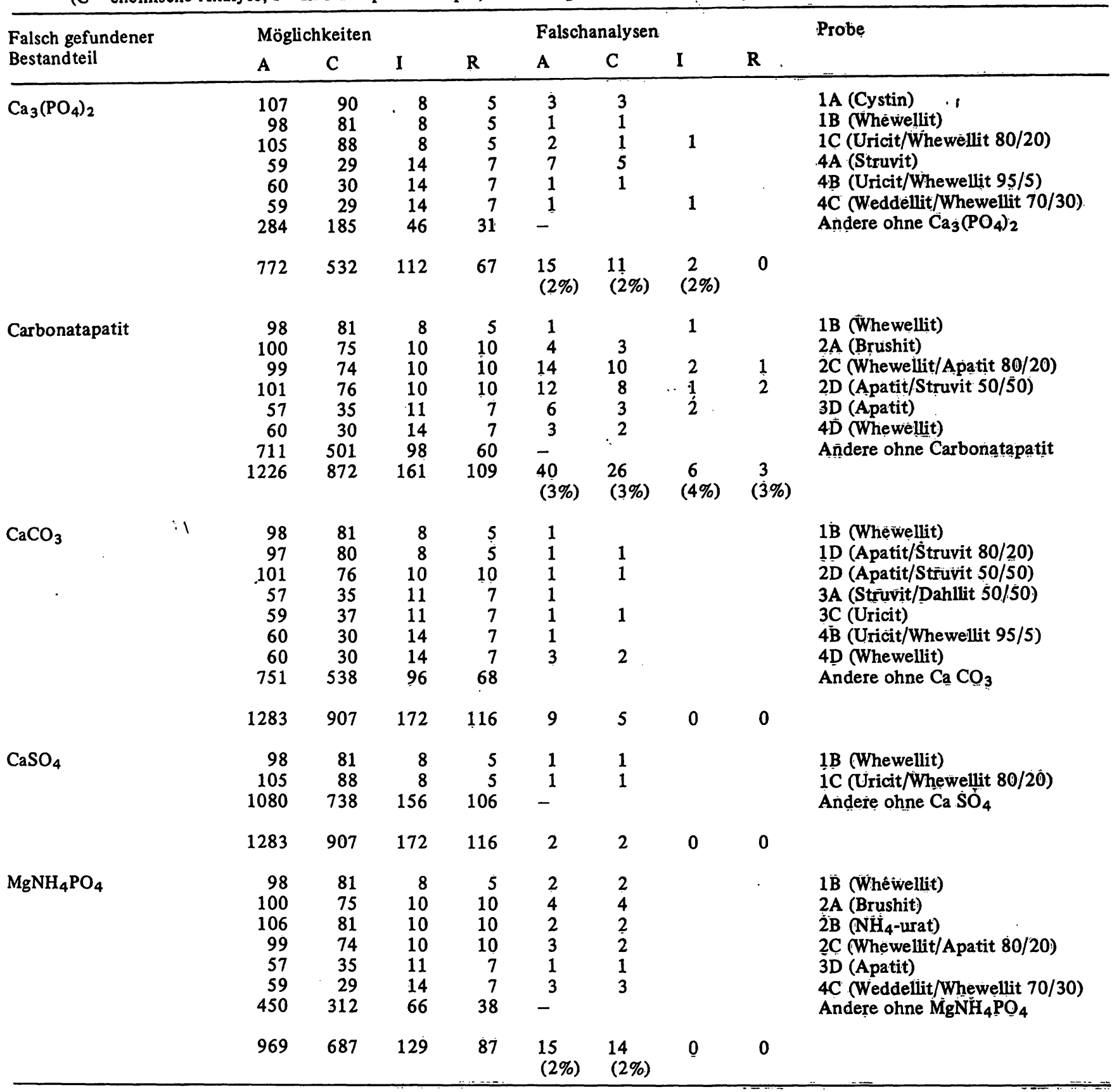

Laboratorium insgesamt durchgeführten Analysen (Abb. 1). Wenn man unterstellt, daß der engagierte Analytiker der Qualitätskontrolle ein besonderes Interesse entgegenbringt, so wären hierdurch die deutlich besseren Ergebnisse aus denjenigen Laboratorien zu erklären, die an allen Ringversuchen teilnahmen: der Anteil von $75-100 \%$ richtiger qualitativer Ergebnisse war in dieser Gruppe fünfmal so hoch wie bei denjenigen, die nur an einem Ringversuch teilgenommen haben.

Abb. 1. Rèlative Anzahl von Laboratorien, deren mit chemischen Methoden durchgefürte qualitative Hamsteinanalysen in den angegebenen Prozentintervallen richtig waren ( $\square$ : Teilnahme an nur einem Ringversuch, 32 Laboratorien; «: Teilnahme an allen vier Ringversuchen, 22 Laboratorien).

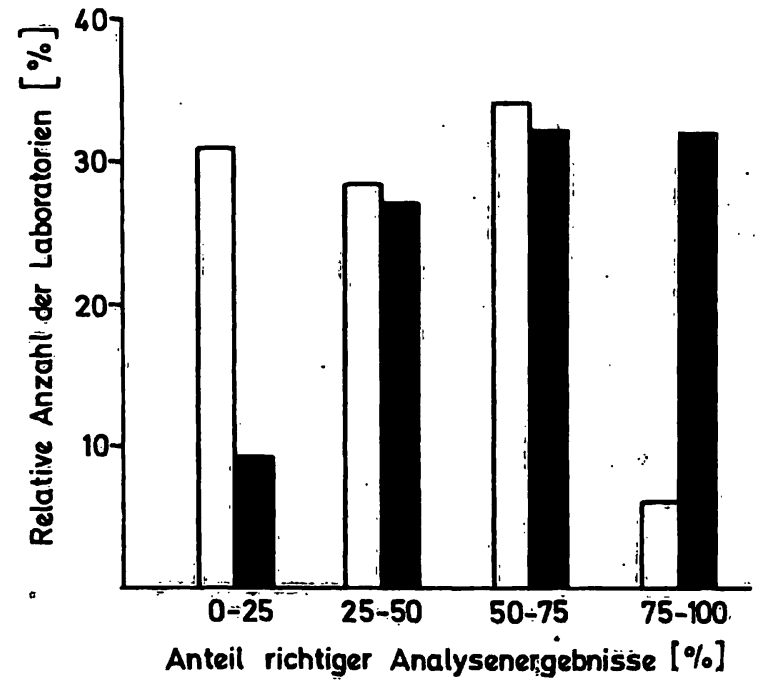

J. Clin. Chem. Clin. Biochem. / Vol. 20, 1982/ No. 12 
Tab. 5 (Fortsetzung)

\begin{tabular}{|c|c|c|c|c|c|c|c|c|c|}
\hline \multirow{2}{*}{$\begin{array}{l}\text { Falsch gefundener } \\
\text { Bestandteil }\end{array}$} & \multicolumn{3}{|c|}{ Möglichkeiten } & \multirow[b]{2}{*}{$\mathbf{R}$} & \multicolumn{3}{|c|}{ Falschanalysen } & \multirow[b]{2}{*}{$\mathbf{R}$} & \multirow[t]{2}{*}{ Probe } \\
\hline & $\mathbf{A}$ & C & 1 & & $\mathbf{A}$ & $\mathrm{C}$ & I & & \\
\hline \multirow[t]{2}{*}{$\mathrm{MgHPO}_{4}$} & $\begin{array}{r}105 \\
97 \\
99 \\
101 \\
881\end{array}$ & $\begin{array}{r}88 \\
80 \\
74 \\
76 \\
589\end{array}$ & $\begin{array}{r}8 \\
8 \\
10 \\
10 \\
136\end{array}$ & $\begin{array}{r}5 \\
5 \\
10 \\
10 \\
86\end{array}$ & $\begin{array}{r}1 \\
1 \\
1 \\
1 \\
-\end{array}$ & $\begin{array}{l}1 \\
1\end{array}$ & $\begin{array}{l}1 \\
i\end{array}$ & & $\begin{array}{l}\text { 1C (Uricit/Whewellit 80/20) } \\
\text { 1D (Apatit/Struvit 80/20) } \\
\text { 2C (Whewellit/Apatit 80/20) } \\
\text { 2D (Apatit/Struvit 50/50) } \\
\text { Andere ohne } \mathrm{MgHPO}_{4}\end{array}$ \\
\hline & 1283 & 907 & 172 & 116 & 4 & 2 & 2 & 0 & \\
\hline \multirow[t]{2}{*}{ Calciumoxalat } & $\begin{array}{r}107 \\
97 \\
100 \\
106 \\
101 \\
57 \\
59 \\
57 \\
59 \\
-\end{array}$ & $\begin{array}{l}90 \\
80 \\
75 \\
81 \\
76 \\
35 \\
37 \\
35 \\
29 \\
-\end{array}$ & $\begin{array}{r}8 \\
8 \\
10 \\
10 \\
10 \\
11 \\
11 \\
11 \\
14 \\
-\end{array}$ & $\begin{array}{r}5 \\
5 \\
10 \\
10 \\
10 \\
7 \\
7 \\
7 \\
7\end{array}$ & $\begin{array}{r}9 \\
11 \\
23 \\
6 \\
14 \\
8 \\
2 \\
11 \\
7 \\
-\end{array}$ & $\begin{array}{r}8 \\
10 \\
23 \\
5 \\
13 \\
8 \\
2 \\
10 \\
6\end{array}$ & 1 & & $\begin{array}{l}\text { 1A (Cystin) } \\
\text { 1D (Apatit/Struvit 80/20) } \\
\text { 2A (Brushit) } \\
\text { 2B (NH } \text { (N-urat) }_{\text {2 }} \\
\text { 2D (Apatit/Struvit 50/50) } \\
\text { 3A (Struvit/Dahllit 50/50) } \\
\text { 3C (Uricit) } \\
\text { 3D (Apatit) } \\
\text { 4A (Struvit) } \\
\text { Andere ohne Ca-oxalat }\end{array}$ \\
\hline & 743 & 538 & 93 & 68 & $\begin{array}{l}91 \\
(12 \%)\end{array}$ & $\begin{array}{l}85 \\
(16 \%)\end{array}$ & $\stackrel{2}{2}$ & 0 & \\
\hline \multirow[t]{2}{*}{ Harnsäure } & $\begin{array}{r}107 \\
98 \\
100 \\
106 \\
99 \\
60 \\
430\end{array}$ & $\begin{array}{r}90 \\
81 \\
75 \\
81 \\
74 \\
30 \\
284\end{array}$ & $\begin{array}{r}8 \\
8 \\
10 \\
10 \\
10 \\
14 \\
69\end{array}$ & $\begin{array}{r}5 \\
5 \\
10 \\
10 \\
10 \\
7 \\
43\end{array}$ & $\begin{array}{r}18 \\
2 \\
2 \\
28 \\
1 \\
1 \\
-\end{array}$ & $\begin{array}{r}18 \\
2 \\
2 \\
24 \\
1 \\
1\end{array}$ & 2 & & $\begin{array}{l}\text { 1A (Cystin) } \\
\text { 1B (Whewellit) } \\
\text { 2A (Brushit) } \\
\text { 2B (NH } 4 \text {-urat) } \\
\text { 2C (Whewellit/Apatit 80/20) } \\
\text { 4D (Whewellit) } \\
\text { Andere ohne Harnsäure }\end{array}$ \\
\hline & 1000 & 715 & 129 & 90 & $\begin{array}{l}52 \\
(5 \%)\end{array}$ & $\begin{array}{l}48 \\
(7 \%)\end{array}$ & $\begin{array}{l}2 \\
(2 \%)\end{array}$ & 0 & \\
\hline \multirow[t]{2}{*}{ Ammoniumurat } & $\begin{array}{r}107 \\
98 \\
105 \\
97 \\
101 \\
59 \\
59 \\
59 \\
60 \\
432\end{array}$ & $\begin{array}{r}90 \\
81 \\
88 \\
80 \\
76 \\
37 \\
37 \\
29 \\
30 \\
278\end{array}$ & $\begin{array}{r}8 \\
8 \\
8 \\
8 \\
10 \\
11 \\
11 \\
14 \\
14 \\
70\end{array}$ & $\begin{array}{r}5 \\
5 \\
5 \\
5 \\
10 \\
7 \\
7 \\
7 \\
7 \\
48\end{array}$ & $\begin{array}{l}3 \\
3 \\
2 \\
2 \\
1 \\
2 \\
4 \\
1 \\
2 \\
-\end{array}$ & $\begin{array}{l}2 \\
3 \\
2 \\
2 \\
4 \\
4 \\
2\end{array}$ & & $\cdot$ & $\begin{array}{l}\text { 1A (Cystin) } \\
\text { 1B (Whewellit) } \\
\text { 1C (Uricit/Whewellit 80/20) } \\
\text { 1D (Apatit/Struvit 80/20) } \\
\text { 2D (Apatit/Struvit 50/50) } \\
\text { 3B (Whewellit/Uricit 60/40) } \\
\text { 3C (Uricit) } \\
\text { 4A (Struvit) } \\
\text { 4B (Uricit/Whewellit 95/5) } \\
\text { Andere ohne NH4-urat }\end{array}$ \\
\hline & 1177 & 826 & 162 & 106 & $\begin{array}{l}20 \\
(2 \%)\end{array}$ & $\begin{array}{c}15 \\
(2 \%)\end{array}$ & 0 & 0 & \\
\hline \multirow[t]{2}{*}{ Natriumiunat } & $\begin{array}{r}105 \\
106 \\
60 \\
1012\end{array}$ & $\begin{array}{r}88 \\
81 \\
30 \\
708\end{array}$ & $\begin{array}{r}8 \\
10 \\
14 \\
140\end{array}$ & $\begin{array}{r}5 \\
10 \\
7 \\
94\end{array}$ & $\begin{array}{r}\frac{2}{1} \\
2 \\
-\end{array}$ & $\begin{array}{l}1 \\
2\end{array}$ & & $\begin{array}{l}1 \\
1\end{array}$ & $\begin{array}{l}\text { 1C (Uricit/Whewellit 80/20) } \\
\text { 2B (NH4-urat) } \\
\text { 4B (Uricit/Whewellit 95/5) } \\
\text { Andere ohne Na-urat }\end{array}$ \\
\hline & 1283 & 907 & 172 & 116 & 5 & 3 & 0 & 2 & \\
\hline \multirow[t]{2}{*}{ Xanthin } & $\begin{array}{r}98 \\
105 \\
106 \\
99 \\
59 \\
60 \\
756\end{array}$ & $\begin{array}{r}81 \\
88 \\
81 \\
74 \\
37 \\
37 \\
30 \\
516\end{array}$ & $\begin{array}{r}8 \\
8 \\
10 \\
10 \\
11 \\
14 \\
111\end{array}$ & $\begin{array}{r}5 \\
5 \\
10 \\
10 \\
7 \\
7 \\
72\end{array}$ & $\begin{array}{l}3 \\
3 \\
3 \\
1 \\
2 \\
1 \\
-\end{array}$ & $\begin{array}{l}3 \\
3 \\
3 \\
1 \\
2 \\
1\end{array}$ & & . & $\begin{array}{l}\text { 1B (Whewellit) } \\
\text { 1C (Uricit/Whewellit 80/20) } \\
\text { 2B (NH } 4 \text {-urat) } \\
\text { 2C (Whewellit/Apatit 80/20) } \\
\text { 3C (Uricit) } \\
\text { 4B (Uricit/Whewellit 95/5) } \\
\text { Andere ohne Xanthin }\end{array}$ \\
\hline & 1283 & 907 & 172 & 116 & 13 & 13 & 0 & 0 & . \\
\hline \multirow[t]{2}{*}{ Cystin } & $\begin{array}{r}98 \\
100 \\
106 \\
101 \\
771\end{array}$ & $\begin{array}{r}81 \\
75 \\
81 \\
76 \\
504\end{array}$ & $\begin{array}{r}8 \\
10 \\
10 \\
10 \\
126\end{array}$ & $\begin{array}{r}5 \\
10 \\
10 \\
10 \\
76\end{array}$ & $\begin{array}{r}1 \\
3 \\
2 \\
1 \\
-\end{array}$ & $\begin{array}{l}1 \\
3 \\
2 \\
1\end{array}$ & & & $\begin{array}{l}\text { 1B (Whewellit) } \\
\text { 2A (Brushit) } \\
\text { 2B (NH4-urat) } \\
\text { 2D (Apatit/Struvit 50/50) } \\
\text { Andere ohne Cystin }\end{array}$ \\
\hline & 1176 & 817 & 164 & 111 & 7 & 7 & 0 & 0 & \\
\hline
\end{tabular}


Besonders gute - auch quantitativ - Resultate wurden in Laboratorien erzielt, in denen - wie sich aus Kommentaren zu den individuellen Analysenergebnissen entnehmen ließ - die üblichen halbquantitativen Methoden der Harnsteinanalyse durch quantitative klinischchemische Bestimmungsmethoden ergänzt werden.

Die Infrarotspektroskopie lieferte insgesamt bessere Ergebnisse als mit chemischen Methoden erreicht wurden. Der Anteil an Fehlanalysen ist jedoch beträchtlich; hauptsächlich waren es Apatit und Whewellit, die nicht identifiziert werden konnten (Tab. 4). Es ist zwar hervorzuheben, daß die Fehlanalysen von Whewellit besonders häufig bei der Probe 4B auftraten, die nur etwa $5 \%$ Whewellit enthielt, aber auch in der Probe $1 \mathrm{C}$ mit $20 \%$ Whewellit gelang es nur sechs von acht Analytikern, diesen Bestandteil nachzuweisen. Ein Teil der Fehlanalysen von Apatit und Whewellit ist wahrscheinlich auf die eingeschränkte Leistungsfähigkeit einiger Geräte zurückzuführen; eine wesentliche Voraussetzung für einwandfreie Analysenergebnisse ist, daß ein Wellenzahlbereich von $400-4000 \mathrm{~cm}^{-1}$ zur Verfügung steht (4).Ergebnis der selteneren Falschanalysen war in erster Linie Carbonatapatit für Proben, die Apatit enthielten (Tab. 5).

Die zuverlässigsten Ergebnisse erbrachte die Röntgendiffraktion. $86 \%$ der qualitativen Analysen führten zum optimalen Analysenziel, d.h. es wurde die genaue chemische Zusammensetzung der Probenbestandteile ohne Falschanalyse bestimmt. Weiterhin hatten $53 \%$ aller mit der Röntgendiffraktion durchgeführten Analysen richtige quantitative Ergebnisse. Immerhin sind auch bei dieser aufwendigen Analysentechnik Fehlinterpretationen nicht ganz zu vermeiden. Neben fünf Fehlanalysen des geringen Whewellitanteils der Probe 4B war es vor allem Apatit, der in Einzelfällen nicht gefunden wurde. Falschanalysen ergaben sich in drei Fällen aus der Verwechslung von Carbonatapatit mit Apatit und in zwei Fällen wurde Natriumurat anstelle von Harnsäure bzw. Ammoniumurat angegeben.

Prinzipiell sind sowohl mit der Infrarotspektroskopie als auch mit der Röntgendiffraktion einwandfreie Analysenergebnisse zu erzielen (5-9). Neben einer ausreichenden technischen Ausstattung der Meßgeräte ist hierzu als weitere Voraussetzung die Verfügbarkeit von Referenzspektren erforderlich, die möglichst mit authentischem Material gerätespezifisch hergestellt oder ersatzweise der Literatur entnommen werden $(4 ; 5)$. Die in den Ringversuchen beobachteten Fehl- und Falschanalysen bei Anwendung der physikalischen Methoden lassen darauf schließen, daß diese notwendigen Voraussetzungen nicht in allen Laboratorien gegeben waren.
Es war das Ziel der beschriebenen Ringversuche, erste Anhaltspunkte über den derzeitigen Stand der Harnsteinanalytik zu erhalten. Abweichend von der Praxis bei der Untersuchung von Biomineralisaten von Patienten wurde - mit Ausnahme der Proben 3A, 4B und 4C synthetisches Material für die Ringversuchsproben verwendet. Aus der Verwendung von synthetischem Material ergab sich der Vorteil, für die Auswertung der Ergebnisse über die gesicherte Kenntnis der tatsächlichen Probenzusammensetzung verfügen zu können; weiterhin war zu erwarten, daß bei vergleichsweise einfachen analytischen Aufgabenstellungen die häufigsten Fehler besser zu èrkennen sind. Andererseits war durch die Homogenisierung des Probenmaterials die Möglichkeit der Analytiker eingeschränkt, aus einer optischen Beurteilung zusätzliche Informationen zu gewinnen.

Soweit es der Modellcharakter von Ringversüchen und der Stand der Harnsteinanalytik zulassen, wird für kïnftige Untersuchungen dieser Art eine weitergehende Ubereinstimmung der Vèrsuchsbedingungen mit den Gegebenheiten der Praxis anzustreben sein.

\section{Schlußfolgerungen}

Nach den vorliegenden Ergebnissen ist der derzeitige Stand der Harnsteinanalytik als unbefriedigend zu bezeichnen. Dies gilt um so mehr als angenommen werden kann, daß die Teilnehmer an den Ringversuchen nicht repräsentativ sind für alle Laboratorien, die Harnsteinanalysen durchführen; es dürfte sich bei den Ringversuchsteilnehmern vielmehr um eine positive Auswahl engagierterer Analytiker handeln.

Insbesondere bei den chemischen Analysen ist die Gefahr offenbar sehr groß, daß dem Analytiker Fehiinterpretationen unterlaufen. Eine Verbesserung der derzeit üblichen Methoden erscheint deshalb dringend notwendig. Auch die grundsätzlich leistungsfähigeren physikalischen Methoden - Infrarotspektroskopie und Röntgendiffraktion - können den an sie zu stellenden Ansprüchen nur dann genügen, wenn die technische Ausstattung der Geräte den Erfordernissen der Harnsteinanalytik entspricht und Referenzdiagramme zur Verfügung stehen.

Aufgrund der Tatsache, daß in den durchgeführten Ringversuchen insgesamt nur $64 \%$ der Analysen das vereinfachte qualitative Analysenziel erreicht haben, stellt sich die Frage, ob die gegenwärtig angewendeten Therapien der Urolithiasis auf einer ausreichend gesicherten Diāgnostik aufgebaut sind. 


\section{Literatur}

1. Uldall, A. (1981) Scand. J. Clin. Lab. Invest. 41, 339-345.

2. Hesse, A., Röhle, G. \& Voigt, U. (1981) in: Pathogenese und Klinik der Harnsteine VIII, pp. 306-310 (Vahlensiek, W. \& Gasser, G., eds.) Wien, Steinkopf Verlag Darmstadt.

3. Vahlensiek, W., Hesse, A. \& Bach, D. (1980) Urologe B 20; 273-276.

4. Hesse, A. \& Bach, D. (1982) Harnsteine - Pathobiochemie und klinisch-chemische Diagnostik. Thieme, Stuttgart.

5. Schneider, H. J. (Herausgeber) (1974) Technik der Harnsteinanalyse. Thieme, Leipzig.
6. Hesse, A. \& Molt, K. (1982) J. Clin. Chem. Clin. Biochem. 20,861-873.

7. Gebhardt, M. \& Bastian, H. P. (1976) Urol. Int. 31, 217-229.

8. Asper, R. \& Schmucki, O. (1980) Therapeutische Umschau 37, 342-347.

9. Rebentisch, G., Berg, W. \& Schneider, H. J. (1981) Z. Med. Labor-Diagn. 22, 324-332.

Dr. G. Röhle

Institut für Klinische Biochemie Sigmund-Freud-Straße 25

D-5300 Bonn 1 
